\title{
An Approximate Noise Computation for General Integrator-Based SC Filters
}

\author{
Josef Goette, Member, IEEE, August Kaelin, Member, IEEE, Walter Guggenbühl, Senior Member, IEEE, \\ and George S. Moschytz, Fellow, IEEE
}

\begin{abstract}
An approximate measure for the noise performance of integrator-based SC-filters of general order $n$ is developed. The measure is dependent only on SC-design parameters like capacitor ratios and capacitance levels, but not on technologydependent parameters describing the switches and amplifiers. A simple and explicit closed-form expression for this approximate noise measure is derived and compared with more complex-and accurate-measures. The comparison demonstrates extraordinary accuracy for our approximate noise computation. An important application for our noise measure is th the optimization of integrator-based SC-filters; it is used in the computer-aided design of sensitivity- and noise-optimized SC filters as described in the companion paper [1].
\end{abstract}

\section{INTRODUCTION}

$\mathrm{T}$ HE main limitation for the application of switchedcapacitor (SC) technology in signal processing tasks is the poor noise performance and the corresponding limited dynamic range of SC filters. Therefore, noise analysis and optimization is crucial. Exact noise computations for SC filters are, however, generally involved and computerintensive. This is not disturbing in an analysis context, where most often only a few noise computations have to be performed. It becomes troublesome, however, when synthesizing SC circuits, because, as mentioned above, dynamic range limitations generally make a design optimization necessary, and this in turn requires many noise analysis runs. It is therefore very desirable to have an easily computable measure that describes the noise performance accurately. Many proposals for the approximate computation of noise in SC circuits have already been made (e.g., [2]-[5]). However, their underlying models lead to noise approximations that are not sufficiently accurate or comprehensive to be interpreted in a more exact modeling environment. They also supply no information about the approximation errors to be expected. Finally, the previously published expressions for noise are

\footnotetext{
Manuscript received February 28, 1990; revised March 1, 1991. This work was supported in part by the Fondation Suisse pour la Recherche en Microtechnique under Grant 5.521.330.553/1. This paper was recommended by Associate Editor M. Ismail.

J. Goette and W. Guggenbühl are with the Electronics Laboratory, Swiss Federal Institute of Technology, CH-8092 Zurich, Switzerland.

A. Kaelin and G. S. Moschytz are with the Institute for Information and Signal Processing, Swiss Federal Institute of Technology, CH-8092 Zurich, Switzerland.

IEEE Log Number 9102877.
}

limited to small building blocks such as integrators or biquads, and cannot be readily generalized to $n$ th-order filters. To overcome these shortcomings, we have developed a new noise measure and derived an explicit closedform expression for its computation for general $n$ th-order intcgrator-based SC filters that use the well-known strayinsensitive two-phase integrators [6] as basic building blocks. These are the most common filters in practical use today. Based on the proposed noise measure, we develop, in a companion paper [1], an efficicnt optimization procedure that minimizes noise for a given capacitance area, and which is implemented in the computer-aided design and optimization tool SCSYN [7]. ${ }^{1}$ The noise performance and dynamic range of SC filters can thus be significantly improved as has been demonstrated by simulation, cf. [1].

The derivation of the new noise measure was stimulated by the following observations regarding noise properties that turn out to be typical for SC filters. Beside their dependence on capacitors, the noise in such circuits is also influenced by the realization of the switches and amplifiers. In their oN-state, the switches can be modeled by finite, fixed conductances $G$ in parallel with white-noise current sources with the (two-sided) spectral density $2 k T G$ ( $k T$ is the product of Boltzmann's constant and the absolute temperature), and in their ofF-state by open circuits. The amplifiers used in SC circuits are mostly of the operational transconductance amplifier (OTA) type, whose broadband noise contribution is modeled by a white-noise current source of spectral density $2 k \operatorname{Tg} \gamma$, with $g$ and $\gamma$ denoting the transconductance and noise factor, respectively. Based on these models, Fig. 1 shows the output noise performance ${ }^{2}$ of a 10th-order bandpass ladder filter ${ }^{3}$ computed by the WATSCAD network analysis package [8]. It illustrates the following properties, which are typical for all the filters we have investigated. The noise variance is linear in the noise factor $\gamma$ and

\footnotetext{
${ }^{1} \mathrm{SCSYN}$ uses the novel noise measure also in structural optinization of biquad cascades in that it allows the fast selection of the best in a class of possible realizations.

${ }^{2}$ Only the predominant discrete-time noise is taken into account [8]

${ }^{3}$ The filter realizes a passband extending from $1.2 \mathrm{kHz}$ to $1.54 \mathrm{kHz}$, a passband ripple of $0.2 \mathrm{~dB}$, and a maximum pole $Q$ of 48 with a clock frequency of $50 \mathrm{kHz}$. We refer to the companion paper [1] for further details and specifications.
} 


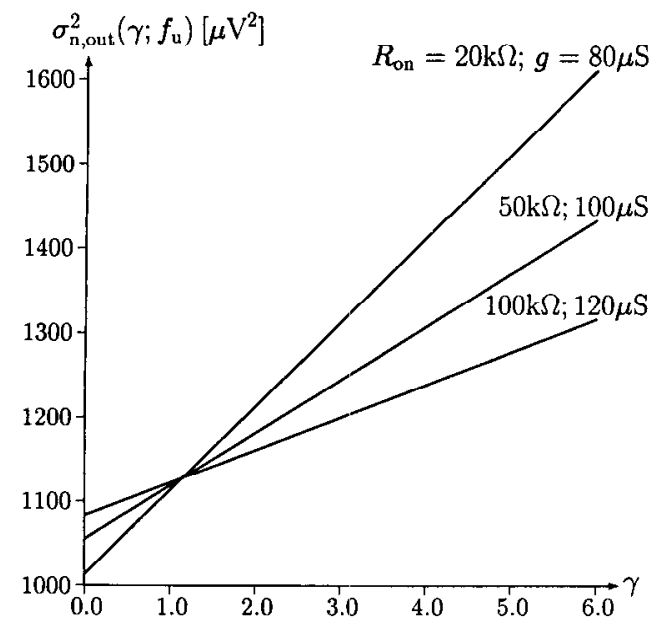

Fig. 1. Noise performance of 10th-order bandpass ladder filter. Noise variance in a $1-\mathrm{Hz}$ band at upper passband edge as a function of the amplifier noise factor $y$ for various switch oN-resistance and amplifier transconductance combinations.

there seems to be a crossing point where all linear characteristics, which differ by $R_{\text {on }}=1 / G$ and $g$, meet. Whereas the linearity in $\gamma$ is found to hold for SC filters in general [9], a true crossing point exists only for undamped SC integrators [10]. For practical SC filters there is, instead of a crossing point, a small region through which the linear characteristics pass. This crossing property is investigated in detail in Section 2.1 for a first-order filter. There we show that the crossing region is, in fact, very small and can be approximated by a point that is exactly defined and designated crossing point. With increasing amplifier transconductance $g$, the noise dependence on $\gamma$ becomes flatter. In the limit, as $g \rightarrow \infty$, the noise is no longer a function of the noise factor $\gamma$ because the amplifier is ideal (infinite transconductance and vanishing noise contribution). Thus the remaining noise is due to switch noise for which the dependence on the switch on-conductance $G$ vanishes as well. This limiting behavior specifies the magnitude of the crossing point and depends only on SC-filter design parameters such as capacitor ratios and capacitance levels, but not on technological parameters describing the switch and amplifier realizations (e.g., $R_{\text {on }}=1 / G$, and $g$ ). Thus the magnitude of the crossing point can serve as a useful measure for the noise performance, which is dependent only on SC-filter design parameters. Whereas this noise measure can efficiently be used to optimize the design parameters of an SC filter [1], the above discussion of the true noise performance can guide the amplifier and switch design.

For the first-order filter mentioned above the introduced noise measure is approximated by a simple closedform expression in Section 2.1. In Section 2.2 we use a signal-flow graph interpretation to postulate the generalization of the derived formula to SC filters of any order. An empirical verification by simulation of the true noise performance is discussed in Section 2.3. The excellent agreement with the simulation demonstrates the usefulness of our noise measure.

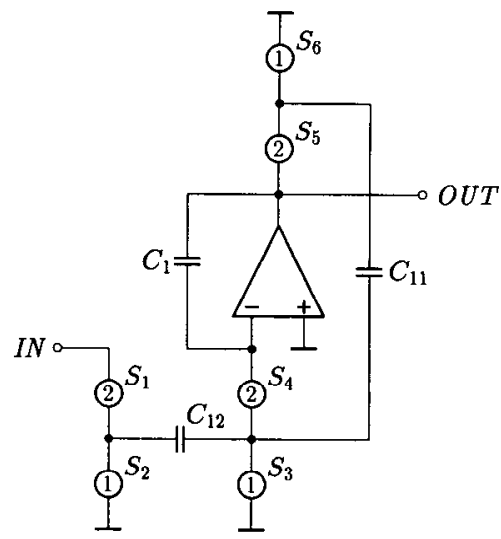

Fig. 2. Circuit diagram of a first-order filter example.

\section{Derivation of the Approximate NOISE Formula}

\subsection{Crossing Point Noise of First-Order Circuit}

'The discrete-time noise in $\mathrm{SC}$ circuits is generated by the interaction of two basic phenomena [8]. One phenomenon involves the sampling of random voltages in each of the noisy continuous-time networks corresponding to the switching phases of the SC filter; the other phenomenon is related to a linear discrete-time filtering of these samples. We shall now investigate these two phenomena using the first-order SC filter in Fig. 2 as an cxample.

Discrete-Time Filtering: For a phase- 2 to phase- 2 operation (i.e., phase 2 at input and output), and taking noise into account, the filter in Fig. 2 is described by ${ }^{4}$

$$
\begin{aligned}
\left(1+k_{11}\right) v_{\text {out }}(n)=v_{\text {out }}(n-1)- & k_{12} v_{\text {in }}(n) \\
& +\Delta_{1}(n)+\Delta_{2}(n)
\end{aligned}
$$

where the voltage samples with index $n$ are taken at the end of phase 2 in the time interval $\left(n T_{s},(n+1) T_{s}\right], T_{s}$ is the sampling period, $k_{11}=C_{11} / C_{1}$ and $k_{12}=C_{12} / C_{1}$ denotc capacitor ratios according to Fig. 2 , and the uncorrelated stationary white noise sequences $\Delta_{1}(\cdot)$ and $\Delta_{2}(\cdot)$ represent the effect of the noise sampled in phase 1 and phase 2 , respectively. The expression in (1) is written such that a signal-flow graph interpretation is possible. This will be required later in Section 2.2. $\Delta_{1}(n)$ and $\Delta_{2}(n)$ are given in terms of the random capacitor-voltage samples $\Delta v_{c j, k}(n)(j \in\{1,11,12\}$ denotes the capacitor index and $k=1,2$ the phase index, respectively) as follows:

$$
\text { Phase 1: } \begin{aligned}
\Delta_{1}(n) \hat{=} & \Delta v_{c 1,1}(n) \\
& -k_{11} \Delta v_{c 11,1}(n)-k_{12} \Delta v_{c 12,1}(n)
\end{aligned}
$$

Phase 2: $\Delta_{2}(n) \hat{=}\left(1+k_{11}\right) \Delta v_{c 1,2}(n)$.

The (co-)variances of the individual capacitor-voltage samples are determined by the continuous-time $R C$ networks realized by the SC circuit in each of its two phases.

\footnotetext{
${ }^{4}$ As usual and justified in practice, full charge transfer is assumed. Furthermore, finite gain effects are neglected.
} 


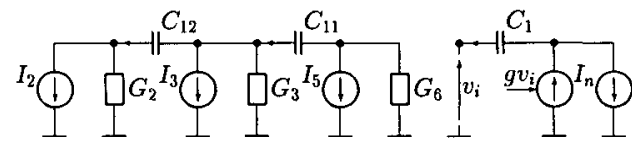

(a)

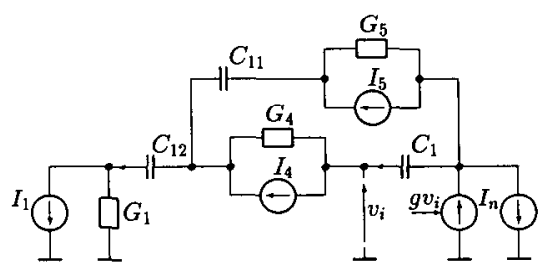

(h)

Fig. 3. Continuous-time circuit diagrams for the noise computation of the first-order SC-filter shown in Fig. 2. (a) Phase 1. (b) Phase 2. The conductances $G_{i}$ correspond to the switches $S_{i}$ and model the active switches together with the white noise current sources $I_{i}$ with spectral density $2 k T G_{i}$. The amplifier noise source $I_{n}$ has a white noise spectral density of $2 k T g \gamma$.

Using the switch and amplifier models, ${ }^{5}$ the resulting networks are shown in Fig. 3(a) (phase 1) and (b) (phase 2).

Phase-1 Noise: The phase-1 network (Fig. 3(a)) shows that there is no current flow through the integrating capacitor $C_{1}$, and consequently no noise generated on this capacitor in phase 1 . Furthermore the capacitors $C_{11}$ and $C_{12}$ are part of a simple passive $R C$ network, which contains neither capacitor-only loops nor capacitor-only cutsets. The detailed analysis given in [11] shows that for this class of circuits the capacitor voltages are uncorrelated with variances of the form $k T / C$. This results here in corresponding variances $k T / C_{11}$ and $k T / C_{12}$, respectively. Thus, according to (2a), the phase-1 induced white noise sequence $\Delta_{1}(\cdot)$ is described by

$$
E\left[\Delta_{1}^{2}\right]=\left(k_{11}+k_{12}\right) \frac{k T}{C_{1}} .
$$

Phase-2 Noise: Fig. 3(b) shows that the phase-2 noise is generated in an active continuous-time $R C$ network. This means that, in this phase, the technology-dependent parameters $G, g$, and $y$ do enter into its description. This dependency, and its characterization by our crossing point, is discussed next. From (2b) it is seen that the phase-2 noise is fully described by the variance of the integrating capacitor voltage, $E\left[\Delta v_{c_{1,2}}^{2}\right]$. This variance may be determined using one of the approaches given in [8]. It is useful to represent the variance in the form

$$
E\left[\Delta v_{c 1,2}^{2}\right]=f(\cdot) \frac{k T}{C_{1}}
$$

where the normalized variance $f(\cdot)$ is a function of the continuous-time circuit parameters $G_{1}, G_{4}$, and $G_{5}$ (which are all equal to the switch on-conductance $G$ ), the amplifier transconductance $g$, the noise factor $\gamma$, and the

\footnotetext{
${ }^{5}$ For the present discussion the OTA is modeled as a voltage-controlled current source in parallel with a white noise current source.
}

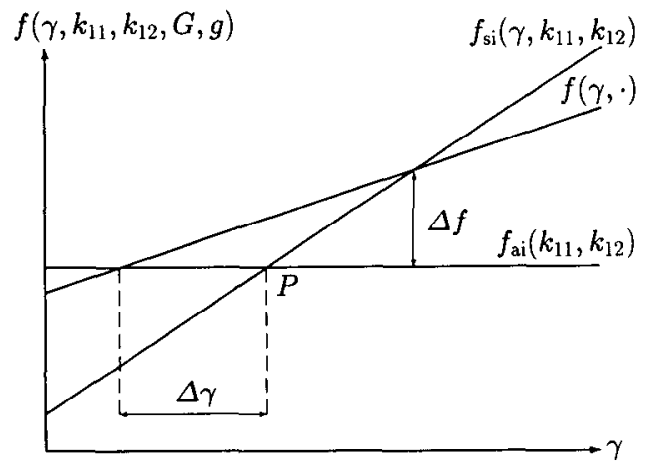

Fig. 4. Normalized integrating capacitor voltage variances $f(\cdot)$ (not true to scale): $f_{\mathrm{ai}}\left(k_{11}, k_{12}\right) \hat{=} \lim _{g \rightarrow \infty} f(\cdot)$ represents the ideal amplifier limiting behavior whereas $f_{\mathrm{si}}\left(\gamma, k_{11}, k_{12}\right)=\lim _{G \rightarrow \infty} f(\cdot)$ symbolizes the ideal switch limiting situation. Also shown is a representative general normalized variance $f(\gamma, \cdot)$ together with the definitions of the displacement variables $\Delta \gamma$ and $\Delta f$

capacitor ratios $k_{11}$ and $k_{12}$. Using a "symbolic mathematics" program [12], it is straightforward to determine $f(\cdot)$, a rather unwicldy cxpression which is, fortunately, not explicitly needed in the following.

In order to obtain an exact definition of the crossing point, we investigate the limiting behavior of $f(\cdot)$ when either the amplifier or the switches are assumed to be ideal. Firstly, the limit of $f(\cdot)$ for an ideal amplifier (i.e., $g \rightarrow \infty)$ is found to be independent of amplifier-induced noise and therefore independent of the noise factor $\gamma$ (as well as independent of $G$ ):

$$
\begin{aligned}
\lim _{g \rightarrow \infty} f(\cdot) & =\frac{2 k_{12}^{2}+\left(2 k_{11}^{2}+4 k_{11}\right) k_{12}+2 k_{11}^{2}}{\left(k_{11}^{2}+3 k_{11}+2\right) k_{12}+2 k_{11}^{2}+2 k_{11}} \\
& \hat{=} f_{a i}\left(k_{11}, k_{12}\right) .
\end{aligned}
$$

This expression has the desirable feature that it depends only on the SC design parameters $k_{11}$ and $k_{12}$, or in other words, it is independent of the noise factor $\gamma$, cf., Fig. 4. Secondly, the limiting behavior as the switches are assumed to be ideal (i.e., the conductances become very large, or $G \rightarrow \infty$ ) leads to (cf., Fig. 4)

$$
\lim _{G \rightarrow \infty} f(\cdot)=\frac{k_{12}}{\left(1+k_{11}\right)^{2}} \gamma+\frac{k_{11}}{1+k_{11}} \hat{=} f_{s i}\left(\gamma, k_{11}, k_{12}\right) \text {. }
$$

Note that beside $\gamma$, this function is again dependent only on the design parameters $k_{11}$ and $k_{12}$.

We now define the intersection of the two limiting characteristics $f_{\mathrm{ai}}(\cdot)$ and $f_{\mathrm{si}}(\cdot)$ to be the crossing point $P$ as indicated in Fig. 4. Unfortunately, with finite switch conductances $G$ and amplifier transconductance $g$, the normalized variance characteristic $f\left(\gamma, k_{11}, k_{12}, G, g\right)$ will not pass exactly through $P$. To describe the deviation from the crossing point $P$, the measures $\Delta \gamma$ and $\Delta f$ are introduced as indicated in Fig. 4. In what follows, we discuss the upper bounds for these measures. For $\Delta \gamma$ corresponding to the circuit in Fig. 2 we find the expression 


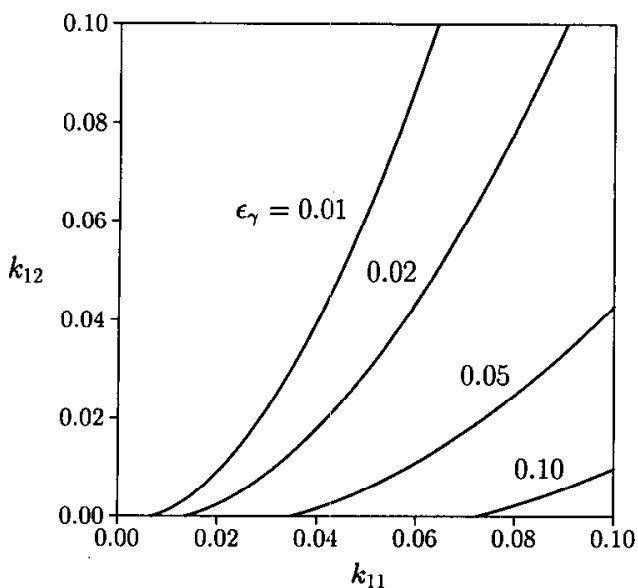

Fig. 5. Contours of the maximum relative deviation $\epsilon_{\gamma}$ in the $k_{11}-k_{12}$ plane for values $0 \leqslant k_{11}, k_{12} \leqslant 0.1$.

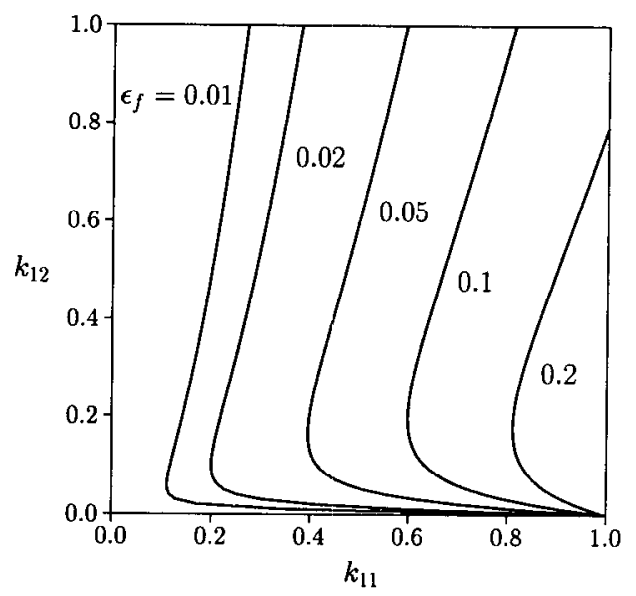

Fig. 6. Contours of the maximum relative deviation $\epsilon_{f}$ in the $k_{11}-k_{12}$ plane for values $0 \leqslant k_{11}, k_{12} \leqslant 1.0$.

$$
\Delta \gamma=\frac{n_{\gamma}\left(k_{11}, k_{12}\right) \cdot g}{d_{g \gamma}\left(k_{11}, k_{12}\right) \cdot g+d_{G \gamma}\left(k_{11}, k_{12}\right) \cdot G}
$$

where $n_{\gamma}(\cdot), d_{g \gamma}(\cdot)$, and $d_{G \gamma}(\cdot)$ are all functions only of $k_{11}$ and $k_{12}$ and are all non-negative. This leads to a non-negative $\Delta \gamma$, which is upper bounded by

$$
\Delta \gamma \leqslant \Delta \gamma_{\operatorname{Indx}} \hat{=} \max _{G, g} \Delta \gamma\left(k_{11}, k_{12}, g, G\right)=\frac{n_{\gamma}\left(k_{11}, k_{12}\right)}{d_{g \gamma}\left(k_{11}, k_{12}\right)} .
$$

The contours of the relative quantity $\epsilon_{\gamma}$ defined by $\epsilon_{\gamma}\left(k_{11}, k_{12}\right) \hat{=} \Delta \gamma_{\max } / \gamma_{P}$ with $\gamma_{P}$ denoting the abscissa of the crossing point $P$ are plotted in the $k_{11}-k_{12}$ plane shown in Fig. 5. This contour plot clearly indicates that the range of the crossing region in the $\gamma$-direction is very small for practical values of $k_{11}$ and $k_{12}$. Analyzing $\Delta_{f}$ in a similar way, the corresponding error quantity $\epsilon_{f}$ is plotted in Fig. 6. Again, the contour plot indicates the small range of the crossing region in the $f$-direction.

Having shown that the linear characteristics of the normalized variance $f(\gamma, \cdot)$ all cross in the close vicinity of the crossing point $P$, we use the magnitude of $P$ as a measure for the variance in (4). With (2b), the corre-

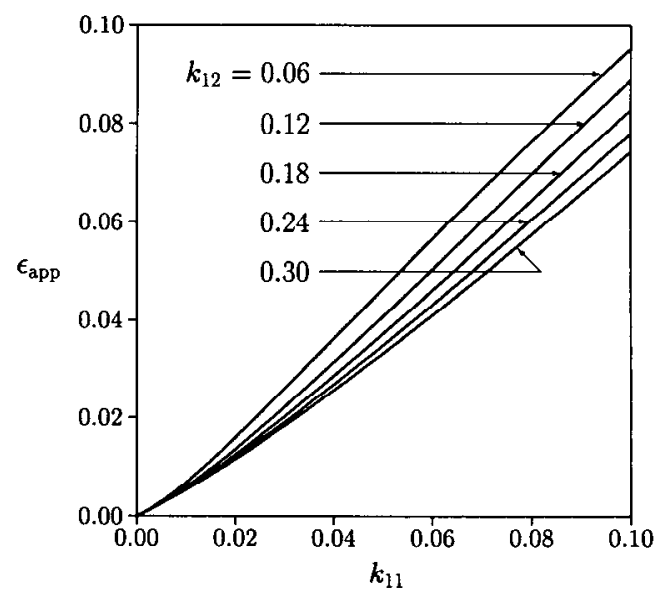

Fig. 7. Relative phase-2 noise approximation error $\epsilon_{\mathrm{app}}\left(k_{11}, k_{12}\right)$ as a function of the feedback capacitor ratio $k_{11}$ for various values of the input capacitor ratio $k_{12}$.

sponding measure for the phase- 2 noise is

$$
E\left[\Delta_{2}^{2}\right]=\left(1+k_{11}\right)^{2} f_{\mathrm{ai}}\left(k_{11}, k_{12}\right) \frac{k T}{C_{1}}
$$

As anticipated, this measure depends only on SC design parameters; see (5). However, the expression is still too complicated to be simply interpreted and generalized for SC circuits of higher complexity. In order to obtain a linear approximation in $k_{11}$ and $k_{12}$, we note that both capacitor ratios are positive and small in practical situations. ${ }^{6}$ Furthermore, since $k_{12}$ is strictly positive in practice (i.e., $k_{12}>0$ ), (5) is defined for all $k_{11} \geqslant 0$. This allows us to approximate $E\left[\Delta_{2}^{2}\right]$ by the linear terms of its Taylor series expansion at $\left(k_{11}, k_{12}\right)=\left(0, k_{120}\right)$ with $k_{120}$ small but positive, i.e.,

$$
E\left[\Delta_{2}^{2}\right] \approx\left(k_{11}+k_{12}\right) \frac{k T}{C_{1}}
$$

Denoting the resulting approximation error by $r\left(k_{11}, k_{12}\right)$, we consider the relative error $\epsilon_{\text {app }}\left(k_{11}, k_{12}\right) \hat{=}$ $r\left(k_{11}, k_{12}\right) / E\left[\Delta_{2}^{2}\right]$ in order to estimate the quality of the above approximation. This relative error is plotted in Fig. 7 as a function of the damping parameter $k_{11}$, for various values of the feed-in parameter $k_{12}$. We find that for $k_{11}$ values in the range $0<k_{11} \leqslant 0.1$, the relative approximation error is less than $10 \%$ for a wide range of $k_{12}$ values. Taking into account the fact that the noise in phase 1 is described by the exact expression (3), the proposed phase2 noise approximation (7) is expected to produce errors of less than about $5 \%$ in the overall noise measure.

\subsection{Signal-Flow Graph Interpretation and Generalization for Higher Order Filters}

Recalling that the noise samples from different phases are uncorrelated, we obtain with (3) and the approxima-

\footnotetext{
${ }^{6}$ This is especially true for high clock frequencies.
} 
TABLE I

Crossing Point Noise of 10th-Order Bandpass Ladder Filter for Various Designs as Computed by WATSCAD and Predicted by OUR Formula (A); OUtPut Noise Variances $\sigma_{n \text {,out }}^{2}$ in a 1-Hz Band at the Filter's Upper Passband EdGe

\begin{tabular}{|c|c|c|c|}
\hline \multirow[b]{2}{*}{ design } & \multicolumn{2}{|c|}{$\sigma_{n, \text { out }}^{2}\left[\mu \mathrm{V}^{2}\right]$} & \multirow{2}{*}{$\begin{array}{c}\text { error } \\
\text { [\%] }\end{array}$} \\
\hline & by WATSCAD & by formula & \\
\hline $5 \mathrm{kHz}$ & 4849.37 & 4709.21 & 2.9 \\
\hline $10 \mathrm{kHz}$ & 2010.47 & 2036.56 & -1.3 \\
\hline $20 \mathrm{kHz}$ & 2024.86 & 2043.86 & -0.94 \\
\hline $50 \mathrm{kHz}$ & 2253.43 & 2262.69 & -0.41 \\
\hline
\end{tabular}

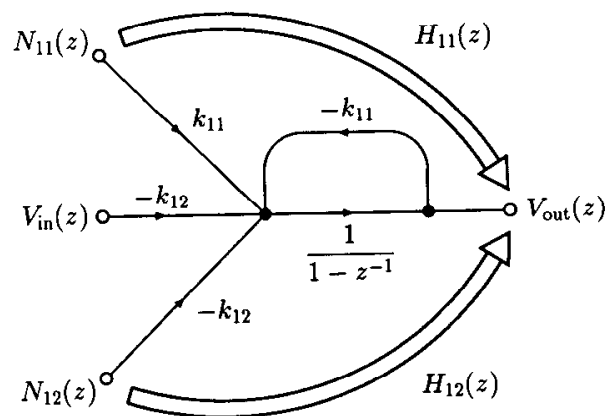

Fig. 8. Signal-flow graph interpretation taking noise into account. $N_{11}(z)$ and $N_{12}(z)$ are the $z$-transforms of the uncorrelated discrete-time noise sources $n_{11}(n)$ and $n_{12}(n)$ respectively, and $H_{11}(z)$ and $H_{12}(z)$ denote the transfer functions from these noise sources to the filter's output.

tion (7) for the variance of the total noise input $\Delta(n) \hat{=}$ $\Delta_{1}(n)+\Delta_{2}(n):$

$$
E\left[\Delta^{2}\right] \approx 2\left(k_{11}+k_{12}\right) \frac{k T}{C_{1}} .
$$

The discrctc-timc filtcring process acting on $\Delta(n)=$ $\Delta_{1}(n)+\Delta_{2}(n)$ according to (1) can now be interpreted with the help of a signal-flow graph. If the noise input is attributed to two uncorrelated white noise sources, $n_{11}(n)$ and $n_{12}(n)$, with variances

$$
E\left[n_{11}^{2}\right]=\frac{2 k T}{k_{11} C_{1}}, E\left[n_{12}^{2}\right]=\frac{2 k T}{k_{12} C_{1}}
$$

the signal flow-graph according to Fig. 8 is obtained. The discrete-time noise spectrum at the filter output is readily derived from this signal-flow graph:

$$
\begin{aligned}
S_{n_{\text {out }}}(\omega)= & \left\{\left|H_{11}\left(\exp \left(j \omega T_{s}\right)\right)\right|^{2} \frac{1}{k_{11}}\right. \\
& \left.+\left|H_{12}\left(\exp \left(j \omega T_{s}\right)\right)\right|^{2} \frac{1}{k_{12}}\right\} \frac{2 k T}{C_{1}} .
\end{aligned}
$$

This expression for the output noise spectrum of a first-order filter suggests the interpretation that the switched capacitors $C_{11}$ and $C_{12}$ each contribute uncorrelated noise samples of variances $2 k T /\left(k_{1 j} C_{1}\right), j=1,2$, to the integrator summing node. Neglecting loading effects we can generalize our noise measure, i.e., (8), for $n$ thorder integrator-based SC filters for which, as mentioned above, a crossing point in the variance characteristic was

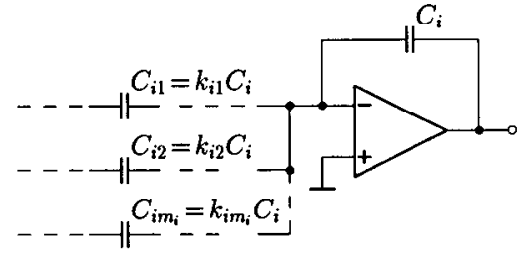

Fig. 9. General stray-intensive integrator stage $i$ and corresponding capacitor notation. The input capacitors $k_{i j} C_{i}$ are arbitrarily connected to their surrounding circuit by phase- 1 and phase- 2 switches or by short circuits.

also observed. We then postulate the following approximate formula describing the discrete-time noise spectrum of the $n$ th-order filter by the crossing point:

$$
S_{n_{\text {out }}}(\omega)=\sum_{i=1}^{n}\left\{\sum_{j \in N_{i}}\left|H_{i j}\left(\exp \left(j \omega T_{s}\right)\right)\right|^{2} \frac{1}{k_{i j}}\right\} \frac{2 k T}{C_{i}} .
$$

Here $n$ denotes the number of integrator stages in the filter, the set $N_{i}$ contains the indices $j$ of the switched input capacitors $C_{i j}$ (i.e., input capacitors connected to their surrounding circuit by switches) of integrator stagc $i$ (cf., Fig. 9), and $H_{i j}(z)$ is the transfer function from capacitor $C_{i j}$ to the filter output.

\subsection{Verification}

We have empirically verified the generalized formula (9) for filters of order $n>1$ for many examples by comparing the crossing point magnitudes predicted by (9) with those oblained from a more exact noise analysis performed by WATSCAD, using the corresponding switch and amplifier models. ${ }^{7}$ For the 10th-order bandpass ladder filter introduced in Section I, four different designs, adapted to their respective clock frequencies, lead to the results summarized in Table $I^{8}{ }^{8}$ It is seen that (9) is quite realistic in describing the crossing point noise for each design. Since we have obtained similar results with numerous other examples, we have reason to believe that (9) is a good choice for the explicit analytic description of the noise performance of an integrator-based SC filter. As

\footnotetext{
${ }^{7}$ We note that the accuracy of WATSCAD noise computations has been verified in [8] by comparing computed and measured results, showing excellent correspondence.

${ }^{8}$ These results are based on a total of $100-\mathrm{pF}$ capacitance assigned to the individual circuit capacitors according to a commonly used procedure [13]. For further details see [1].
} 
such, it is well suited for the performance optimization of $\mathrm{SC}$ filters with regard to dynamic range and signal-to-noise ratio, as proposed in a companion paper [1].

\section{Conclusions}

A measure for the noise performance of two-phase integrator-based SC-filters has been developed. Such SC filters are the most common in practical use today. The measure depends only on SC-design parameters, i.e., it is independent of technology parameters such as switch ON-conductances, amplifier transconductances, and noise factors. A simple closed-form expression has been derived that accurately estimates the noise measure and is well suited for use in SC-filter design, where it permits noise performance optimization in analytical form [1]. Furthermore, in a more accurate noise modeling context involving also the technology-dependent parameters, it permits design optimization also of the amplifiers and switches of SC filters. The expression for the noise measure is sufficiently concise and analytical as to be usable also in $\mathrm{SC}$-filter design based on symbolical computations. A generalization of the proposed noise measure for other types of SC filters, such as FIR structures and time-multiplexed filters, and to low-frequency dominant noise (e.g., flicker noise), is currently under investigation.

\section{REFERENCES}

[1] A. Kaelin, J. Goette, W. Guggenhïhl and G. S. Moschytz, "A novel capacitance assignment procedure for the design of sensitivity- and noise-optimized SC-filters," IEEE Trans. Circuits Syst., Nov. 1991.

[2] B. Furrer and W. Guggenbühl, "Noise analysis of sampled-data circuits," Archiv f. Elektronik u. Ubertragungstechnik, vol. 35, pp. 426-430, 1981.

[3] F. Maloberti, F. Montecchi, and V. Svelto, "Noise and gain in a SC integrator with real operational amplifier," Alta Frequenza, pp. $4-11,1981$.

[4] C.-A. Gobet and A. Knob, "Noise analysis of switched capacitor networks," IEEE Trans. Circuits Syst., vol. CAS-30, pp. 37-43, Jan. 1983.

[5] F. Krummenacher, "Optimization des filters à capacités commutées à très faible consommation, Ph.D. dissertation, Ecole Polytechnique Fédérale de Lausanne, Lausanne, Switzerland, 1985.

[6] K. W. Martin, "Improved circuits for the realization of switchedcapacitor filters," IEEE Trans. Circuits Syst., vol. CAS-27, pp. 237-244, Apr. 1980.

[7] A. Kaelin and Arnold Muralt, "LADNET and SCSYN-SC-filter design tools," Tech. Rep. 8914, Institute for Signal and Information Processing, Swiss Federal Institute of Technology, Zurich, 1989.

[8] J. Goette and C.-A. Gobet, "Exact noise analysis of SC-circuits and an approximate computer implementation," IEEE Trans. Circuits Syst., vol. 36, pp. 508-521, Apr. 1989.

[9] J. Goette and A. Kaelin, "Criteria and methods for the selection of optimized capacitor values in SC-filters," Tech. Rep. 88/6, Electronics Laboratory, Swiss Federal Institute of Technology Zurich, Zurich, Switzerland, 1988.

[10] J. Gocttc and W. Guggenbühl, "Noise performance of SC-integrators assuming different operational transconductance amplifier (OTA) models," IEEE Trans. Circuits Syst., vol. 35, pp. 1042-1048, Aug. 1988.
[11] J. Goette, "The capacitor-voltage variance matrix and passive thermal-noisy $K C$-networks," IEEE Trans. Circuits Syst., vol. 37, pp. 954-959, July 1990 .

[12] Computer-Aided Mathematics Group, Symbolics, Inc. MACSYMA Reference Manual, Version 12, 1986.

[13] R. Gregorian and G. C. Temes, Analog MOS Integrated Circuits for Signal Pracessing. Wiley: New York, 1986.

Josef Goette (S'83--M'91), for a photograph and biography, please see page 1179 of the October issue of this TRANSACtions.

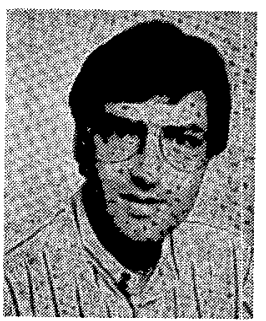

August Kaelin ( $\mathrm{S}^{\prime} 83-\mathrm{M}^{\prime} 91$ ) received the diploma and the Dr. Sc. Tech. degrees in electrical engineering from the Swiss Federal Institute of Technology in 1983 and 1990, respectively.

In 1983, he joined the Institute for Signal and Information Processing at ETH as a teaching assistant. Since 1986 he has been a Research Associate. His current research interests include various aspects of switched-capacitor, digital, and adaptive filters.

Walter Guggenbühl (SM'60), for a photograph and biography, please see page 1179 of the October issue of this Transactions.

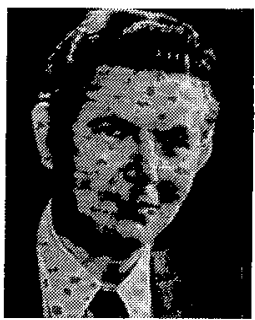

George S. Moschytz (M'65-SM'75-F'78) received the M.S. and Ph.D. degrees in electrical engineering from the Swiss Federal Institute of Technology, Zurich, Switzerland, in 1958 and 1962, respectively.

From 1960 to 1962 he was with the RCA Laboratories Ltd., Zurich, Switzerland. In 1963 he joined Bell Telephone Laboratories, Inc., Holmdel, NJ. Since 1973 he has been Professor of Electrical Engineering and Director of the Institute for Signal and Information Processing at the Swiss Federal Institute of Technology. He is author of Linear Integrated Networks: Fundamentals (New York: Van Nostrand Reinhold, 1974), Linear Integrated Networks: Design (New York, Van Nostrand Reinhold, 1975), co-author of the Active Filter Design Handbook (London: Wiley, 1981), and editor of MOS Switched-Capacitor Filters: Analysis and Design (New York: IEEE Press, 1984). He has authored numerous papers in the field of network theory, design, and sensitivity, and holds several patents in these areas. His present interests are in the fields of digital, switched-capacitor, and adaptive filters, neural networks for signal processing, and the application of signal processing techniques to biological systems.

Dr. Moschytz is president of the IEEE Swiss Chapter on Digital Communication Systems and a member of the Swiss Electrotechnical Society. From 1981 to 1982 he was President of the Swiss Section of the IEEE. He has held several terms in the Adcom of the IEEE Circuits and Systems Society as well as on the Editorial Board of the Proceedings of the IEEE and has been associate editor of the IEEE Circuits and Systems Magazine. $\mathrm{He}$ is also an associate editor of several other technical journals. $\mathrm{He}$ is an elected member of the Swiss Academy of Engineering Sciences and a member of the Eta Kappa Nu Honor Society. 